\title{
Normative Kidneys Measurements in Sudanese: A Morphometric CT Based Study
}

\author{
Afaf Mohamed Ahmed Medani ${ }^{1}$,Caroline Edward Ayad ${ }^{1}$, Mohmmed Elfadil \\ Mohammed Garelnabi ${ }^{1}$. Hussein Ahmed Hassan ${ }^{1}$ \\ ${ }^{I}$ College Of Medical Radiological Science, Sudan University Of Science And Technology, Khartoum, Sudan, \\ P.O.Box 1908
}

\begin{abstract}
:
Background: Normal values for kidney measurements are well established for many ethnic groups but very little is known about normal kidney measurements and its influencing factors in Sudanese. The objectives of this study were to establish a national reference for normal renal dimensions classified according to age and gender for Sudanese as well as to identify potential influencing factors, and to estimate their significance.

Methods: In multiphase thin-slice MDCTs of 200 kidneys in 100 Sudanese subjects, the kidney length and kidney width were registered. The renal parenchymal thickness (PT) including on CT scans was measured through upper calyx; renal pelvis; and lower calyx levels. PT was measured at four locations: anterior (a), posterior $(p)$, medial $(c)$, and lateral $(d)$, at right angles to each other and oriented such that a-b paralleled the renal vessels. The possible influencing factors that can be visualized were recorded from a measurement data set. For measurements, axes were adjusted individually in axial planes. Analyses of distribution, T-tests, ANOVA, were performed.

Results: Kidney length was $96.75 \mathrm{~mm}$ for the right, and $95.73 \mathrm{~mm}$ for the left. The right kidneys width was 41.00 $\mathrm{mm}$, less than the left $42.51 \mathrm{~mm}$ at $(p=0.041)$. The cortex width was $5.21 \mathrm{~mm}$ for the right and $5.26 \mathrm{~mm}$ for the left. While the medulla was $10.82 \mathrm{~mm}$ for the right kidney and $11.21 \mathrm{~mm}$ for the left. Segments as anterior, posterior, medial and lateral segments were measured. Right upper calyx were found to be for $11.88 \mathrm{~mm}$, $16.21 \mathrm{~mm}, 11.74 \mathrm{~mm}$ and $15.56 \mathrm{~mm}$ and the left were $2.00 \mathrm{~mm}, 16.11 \mathrm{~mm}, 12.99 \mathrm{~mm}, 15.52 \mathrm{~mm}$ respectively .The $R T$ renal pelvis segments measurements were found to be $0.00 \mathrm{~mm}, 22.28 \mathrm{~mm}, 13.41 \mathrm{~mm}$ and14.33 $\mathrm{mm}$ where the LT renal pelvis were $0.00 \mathrm{~mm}, 22.37 \mathrm{~mm}, 13.40 \mathrm{~mm}$ and $14.02 \mathrm{~mm}$. The lower calyx segments were found to be 19.45 $\mathrm{mm}, 18.40 \mathrm{~mm}, 13.15 \mathrm{~mm}$ and $13.35 \mathrm{~mm}$ for the right and $10.88 \mathrm{~mm}, 18.85 \mathrm{~mm}, 12.45 \mathrm{~mm}$ and12.29 $\mathrm{mm}$ for the left. The gender is not a predictor for changes in the renal measurements except for the medial and lateral renal pelvis segments for both right and left kidneys at $(P=0.041,0.040$ and 0.001, 0.040). The age also is not a predictor for renal measurement except the renal pelvis lateral segment of the left kidney as well as lower calyx medial segment at $p=0.006$ and0.026. Sudanese kidneys measurements differs from other populations.

Conclusions: A new chart for renal character and measurement were established for Sudanese population aged from 10 years to 70 years
\end{abstract}

Keywords : - CTscan, renal parenchyma, Measurements, Ethnicity

\section{Introduction}

A normal human being has two kidneys. Kidneys filter the waste products from the blood and excrete them out in the form of urine. A normal functioning kidney should have normal renal volume specific for that particular gender and ethnicity. Evaluation of renal measurements such as length, width is an important parameter in the diagnosis and also management in many renal disorders as it is known that there is a close relationship between renal size and its function [1].The evaluation of renal measurements is very important to the clinician as the results can be used to determine the health of the individuals and it can also visualize any abnormalities present in the kidneys. [2] Medical imaging has played an important role in helping physicians to make a medical diagnosis . These imaging included ultrasound and computed tomography (CT) scan. [3] CT scan is extensively used for diagnosing and evaluating kidney problems. One of the parameters which is measured and evaluated in CT scan reports is the kidney length. So, it is important to know the correct calculated length.[5]

One of the important factors in the evaluation of urogenital system is its size. Renal length measurement has a special place in the diagnosis and treatment of renal diseases. Studies conducted have shown that in various diseases, the kidney size changes, which are probably due to inflammation, diabetes and chronic diseases. [4] Symptom of chronic renal failure is increase in kidney size; also the first sign of diabetic nephropathy is the change in kidney size.[5]Kidney size increased in renal failure; furthermore, a kidney which has a double collecting system is usually long. [6,7] Renal ischemia also causes unilateral size change.[8] Therefore, controlling the size of kidneys and comparing them can be a major criterion in detection of renal damages.[6] In research conducted in 2009, Goldny et al. introduced the size of kidney as a criterion for the 
health of adult kidneys.[9]Many studies have shown that the renal size and measurements is influenced by many factors such as age, ethnicity, gender, weight and height $[10,11,12]$. It is also known that the left kidney is larger than the right kidney, independent of gender $[13,14,15]$. Many studies also concluded that renal measurements variation occurs in nephropathies. $[13,16]$

One study found that the most important measurement of renal size is longitudinal length in subjects with normal renal function [3]. Thus, it is essential to establish the pattern of renal measurements for a more accurate diagnosis. Therefore, the kidney size can be suggested as a criterion for kidney health. So, obtaining the true measurements of kidney can be helpful in various diagnoses, and one of the main methods for finding the kidney size is through CT scan.

In practice, measurements of renal size of any age are compared with the measurements that are predicted by standard nomograms. However, to our best knowledge, the current nomograms which are widely used locally were derived from studies based on western population of relatively small sample sizes [17]. Since our currently used renal nomogram in Sudanese is based on the western database, it might lead to the false positive and false negative diagnosis of kidney condition. A wrong diagnosis on a normal kidney will end up with repeated unnecessary scans and patient's anxiety. Therefore, there is an urge to develop specific ethnic (for instance: Sudanese ethnic) population nomogram to provide a better accuracy of renal measurements in terms of making a proper medical diagnosis and also during monitoring the disease progress. Thus, this research is conducted as a first step in order to formulate a nomogram of renal measurements for Sudanese population.

\subsection{Materials:}

\section{Materials And Methods}

Neusoft multi-slice CT Scanner System Model: NeuViz 128, Volt: 3N-380/400v, Power: 90KVA (120 $\mathrm{kV}, 40 \mathrm{MA}$ ) (5 mm slice) in complex of general Omer Sawi was performed, Antalya medical center (5 mm slice) and modern medical center.

\subsection{Population of the study:}

The population of this study consisted of normal kidneys free from any pathology,with normal renal function. The study includes both genders with their age ranged from 10 years to $70 y e a r s$ old.

\subsection{Design of the study:}

This study is analytic study of a case control type deals with CT scan.

\subsection{Place and duration of the study:}

The study population consisted of patients who referred to the CT scan department for CT abdomen; each patient was examined with CT (MDCT) scan by a qualified technologist. Between August 2014 to August 2016 in Sudan at (ALRabat National Hospital, Omdurman Hospital, Modern Medical Center, and Antalya Medical Center

\subsection{Methods of data analysis:}

Methods were used: axial, coronal single image, coronal multi image 100 patients referred for abdomen CT scans were included in the study. Patients both kidneys were investigated . Multi-slice CT was used in this research because by the obtained coronal and axial cuts, the actual length of kidney can be obtained easily. After that CT images were stored in computer disk were viewed by the Radiant, DICOM viewer Digital imaging and communication on medical in computer to selected the coronal images that suit the criteria of research population then uploaded into the computer based software Interactive Data Language (IDL) Then the image were read by IDL the first order were extracted from Multi-slice CT A non contrast to obtain coronal, Axial and sagittal cuts, to measure the renal parenchymal thickness (PT) on CT scans through A, upper calyx; B, renal pelvis; and C, lower calyx levels. PT was measured at four locations: anterior (a), posterior (b), medial (c), and lateral (d), at right angles to each other and oriented such that a-b paralleled the renal vessels. The length and the width of the kidneys, CT No of cortex and medulla, was also measured. And also Multi-slice CT with contrast was used to obtain Axial cuts, to measure the cortex and medulla.

\subsection{Methods of data collection \\ 2.6.1Technique}

CT scans were performed including protocol of axial images from the xiphoid process covers all abdominal area and pelvic down to pubic bone with patient in supine position, head first. The images were made at $100 / 120 \mathrm{kV}$ and $60 / 80 \mathrm{MAs}$, with $5 \mathrm{~mm}$ slice thickness reformat $1.2 \mathrm{~mm}$. Reconstruction used $5 \mathrm{~mm}$ to obtain coronal views. A light diet for $6 \mathrm{hr}$ was preparation for patients. The simultaneous acquisition of multiple thin 
collimated slices in combination with enhanced gantry rotation speed offers thin slice coverage of extended volumes without any loss in spatial resolution.

CT scans of the urinary tract were performed with and/or without intravenous iodinated contrast material depending on the indications. With rapid Scanning and contrast bolus timing, several sequential phases of pacification within the kidney was delineated .The corticomedullary phase was seen if scanning is performed during the first 20 to 90 seconds after contrast administration and represents the early preferential blood flow to the renal cortex . Subsequently, contrast begins to pass into the distal collecting tubules within the renal medulla, resulting in a more homogeneous opacification of the renal parenchyma, termed the CT nephrographic phase. This generally occurs around 2 to 4 minutes after contrast medium injection. Finally, the excretory phase is seen when contrast opacifies the collecting system. Each different disease processes and thus various scanning protocols are used to evaluate the kidneys depending on the indication.

\subsection{Ethical approval}

The ethical approval was granted from the radiology departments; which include commitment of no disclose any information concerning the patient identification as well as consent from the patients. Informed consent was obtained from every patient prior to the examination.

\section{Results}

Table 1: Descriptive Statistics of kidneys characteristics data and CT (HU) for cortex and medulla for both right and left kidneys and T-Test.

\begin{tabular}{|c|c|c|c|c|c|}
\hline & Side & $\mathbf{N}$ & Mean & Std. Deviation & P-value \\
\hline \multirow{2}{*}{ Length Of Kidney } & Left & 100 & 95.73 & 11.40 & 0.495 \\
\cline { 2 - 5 } & Right & 100 & 96.75 & 9.61 & \\
\hline \multirow{2}{*}{ Width Of Kidney } & Left & 100 & 42.51 & 5.15 & \multirow{2}{*}{0.041} \\
\cline { 2 - 5 } & Right & 100 & 41.00 & 5.24 & \\
\hline \multirow{2}{*}{ Cortex(Axial) } & Left & 100 & 5.26 & 0.82 & 0.633 \\
\cline { 2 - 5 } & Right & 100 & 5.21 & 0.82 & \\
\hline \multirow{2}{*}{ Medulla(Axial) } & Left & 100 & 11.21 & 1.68 & \multirow{2}{*}{0.125} \\
\cline { 2 - 5 } & Right & 100 & 10.82 & 1.84 & \\
\hline
\end{tabular}

Table2: Descriptive Statistics of the both kidneys measurements at the upper calyx, renal pelvis, Lower calyx measured at all directions and T-Test.

\begin{tabular}{|c|c|c|c|c|c|c|c|c|c|c|}
\hline & \multicolumn{4}{|c|}{ Upper Calyx } & \multicolumn{3}{|c|}{ Renal Pelvis } & \multicolumn{3}{|c|}{ Lower Calyx } \\
\hline & Side & Mean & Std. V & $\begin{array}{c}\mathrm{P}- \\
\text { value }\end{array}$ & Mean & Std. V & $\begin{array}{c}\mathrm{P}- \\
\text { value }\end{array}$ & Mean & Std. V & $\begin{array}{c}\mathrm{P}- \\
\text { value }\end{array}$ \\
\hline \multirow{2}{*}{$\begin{array}{l}\text { Anterior } \\
\text { (a) }\end{array}$} & Left & 12.00 & 2.60 & \multirow[t]{2}{*}{0.771} & 0.00 & 0.00 & \multirow[t]{2}{*}{-} & 10.88 & 2.44 & \multirow[t]{2}{*}{0.357} \\
\hline & Right & 11.88 & 3.14 & & 0.00 & 0.00 & & 19.45 & 92.32 & \\
\hline \multirow{2}{*}{$\begin{array}{c}\text { Posterior( } \\
\text { p) }\end{array}$} & Left & 16.11 & 3.95 & \multirow[t]{2}{*}{0.847} & 22.37 & 3.63 & \multirow[t]{2}{*}{0.859} & 18.85 & 3.85 & \multirow[t]{2}{*}{0.431} \\
\hline & Right & 16.21 & 4.09 & & 22.28 & 3.65 & & 18.40 & 4.10 & \\
\hline \multirow{2}{*}{ Medial (c) } & Left & 12.99 & 2.85 & \multirow[t]{2}{*}{0.001} & 13.40 & 3.09 & \multirow[t]{2}{*}{0.981} & 12.45 & 3.09 & \multirow[t]{2}{*}{0.146} \\
\hline & Right & 11.74 & 2.39 & & 13.41 & 2.43 & & 13.15 & 3.61 & \\
\hline \multirow{2}{*}{ Lateral (d) } & Left & 15.52 & 3.92 & \multirow{2}{*}{0.941} & 14.02 & 2.99 & \multirow{2}{*}{0.507} & 12.29 & 2.70 & \multirow{2}{*}{0.018} \\
\hline & Right & 15.56 & 3.80 & & 14.33 & 3.64 & & 13.35 & 3.51 & \\
\hline
\end{tabular}

Table 3: WE Statistics of the Kidneys length, width, cortex width and medulla width and CT(HU) and ANOVA

\begin{tabular}{|c|c|c|c|c|c|c|c|c|c|c|}
\hline \multicolumn{11}{|c|}{ Test } \\
\hline \multirow{2}{*}{ 总 } & \multirow{2}{*}{ 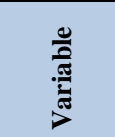 } & \multirow{2}{*}{ : } & \multirow[t]{2}{*}{$\mathbf{N}$} & \multirow[t]{2}{*}{ Mean } & \multirow[t]{2}{*}{$\begin{array}{c}\text { Std. } \\
\text { V }\end{array}$} & \multicolumn{2}{|c|}{$\begin{array}{l}95 \% \text { Confidence } \\
\text { Interval for Mean }\end{array}$} & \multirow[t]{2}{*}{ Min } & \multirow[t]{2}{*}{ Max } & \multirow[t]{2}{*}{$\begin{array}{c}\text { P- } \\
\text { value }\end{array}$} \\
\hline & & & & & & & & & & \\
\hline \multirow{11}{*}{ Left } & \multirow{3}{*}{$\begin{array}{l}\text { length of } \\
\text { kidney }\end{array}$} & Male & 42 & 95.00 & 11.28 & 91.49 & 98.52 & 71.30 & 117.80 & \multirow[t]{3}{*}{.591} \\
\hline & & Female & 58 & 96.25 & 11.55 & 93.21 & 99.29 & 65.30 & 123.40 & \\
\hline & & Total & 100 & 95.73 & 11.40 & 93.47 & 97.99 & 65.30 & 123.40 & \\
\hline & \multirow{3}{*}{$\begin{array}{l}\text { width of } \\
\text { kidney }\end{array}$} & Male & 42 & 42.27 & 5.24 & 40.64 & 43.91 & 31.50 & 54.70 & \multirow[t]{3}{*}{.696} \\
\hline & & Female & 58 & 42.68 & 5.11 & 41.34 & 44.03 & 33.20 & 57.60 & \\
\hline & & Total & 100 & 42.51 & 5.15 & 41.49 & 43.53 & 31.50 & 57.60 & \\
\hline & \multirow{3}{*}{$\begin{array}{l}\text { Cortex } \\
\text { (axial) }\end{array}$} & Male & 41 & 5.37 & 0.87 & 5.10 & 5.65 & 3.20 & 6.85 & \multirow[t]{3}{*}{.282} \\
\hline & & Female & 58 & 5.19 & 0.79 & 4.98 & 5.40 & 3.10 & 6.70 & \\
\hline & & Total & 99 & 5.26 & 0.82 & 5.10 & 5.43 & 3.10 & 6.85 & \\
\hline & \multirow{2}{*}{$\begin{array}{c}\text { Medulla } \\
\text { (axial) }\end{array}$} & Male & 42 & 11.15 & 1.34 & 10.73 & 11.57 & 7.60 & 15.00 & \multirow[t]{2}{*}{.762} \\
\hline & & Female & 58 & 11.25 & 1.90 & 10.75 & 11.75 & 6.80 & 17.60 & \\
\hline
\end{tabular}


Normative Kidneys Measurements In Sudanese: A Morphometric CT Based Study

\begin{tabular}{|c|c|c|c|c|c|c|c|c|c|c|}
\hline & & Total & 100 & 11.21 & 1.68 & 10.87 & 11.54 & 6.80 & 17.60 & \\
\hline \multirow{12}{*}{ Right } & \multirow{3}{*}{$\begin{array}{l}\text { length of } \\
\text { kidney }\end{array}$} & Male & 42 & 95.11 & 8.61 & 92.43 & 97.79 & 79.60 & 113.40 & \multirow[t]{3}{*}{.148} \\
\hline & & Female & 58 & 97.94 & 10.19 & 95.26 & 100.62 & 75.30 & 121.90 & \\
\hline & & Total & 100 & 96.75 & 9.61 & 94.8 & 98.66 & 75.30 & 121.90 & \\
\hline & \multirow{3}{*}{$\begin{array}{l}\text { width of } \\
\text { kidney }\end{array}$} & Male & 42 & 41.76 & 5.76 & 39.97 & 43.56 & 31.70 & 55.90 & \multirow[t]{3}{*}{.219} \\
\hline & & Female & 58 & 40.45 & 4.81 & 39.19 & 41.72 & 30.00 & 51.90 & \\
\hline & & Total & 100 & 41.00 & 5.24 & 39.96 & 42.04 & 30.00 & 55.90 & \\
\hline & \multirow{3}{*}{$\begin{array}{l}\text { Cortex } \\
\text { (axial) }\end{array}$} & Male & 42 & 5.27 & 0.87 & 4.99 & 5.54 & 3.30 & 6.90 & \multirow[t]{3}{*}{.554} \\
\hline & & Female & 57 & 5.17 & 0.7 & 4.95 & 5.38 & 3.20 & 6.70 & \\
\hline & & Total & 99 & 5.21 & 0.82 & 5.04 & 5.37 & 3.20 & 6.90 & \\
\hline & \multirow{3}{*}{$\begin{array}{l}\text { Medulla } \\
\text { (axial) }\end{array}$} & Male & 42 & 11.18 & 1.85 & 10.61 & 11.76 & 7.10 & 14.10 & \multirow{3}{*}{.093} \\
\hline & & Female & 58 & 10.55 & 1.80 & 10.07 & 11.03 & 5.40 & 14.80 & \\
\hline & & Total & 100 & 10.82 & 1.84 & 10.45 & 11.19 & 5.40 & 14.80 & \\
\hline
\end{tabular}

Table 4: Descriptive Statistics of the Kidneys measurements at upper calyxes distributed according to gender and ANOVA test

\begin{tabular}{|c|c|c|c|c|c|c|c|c|c|c|}
\hline 总 & 产育 & 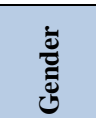 & $\mathbf{N}$ & Mean & $\begin{array}{c}\text { Std. } \\
\text { V }\end{array}$ & \multicolumn{2}{|c|}{$\begin{array}{c}\text { 95\% Confidence } \\
\text { Interval for } \\
\text { Mean } \\
\end{array}$} & Min & $\operatorname{Max}$ & $\begin{array}{c}\text { P- } \\
\text { value }\end{array}$ \\
\hline \multirow{12}{*}{ Left } & \multirow{3}{*}{$\begin{array}{l}\text { Anterior } \\
\text { (a) }\end{array}$} & Male & 42 & 12.05 & 2.83 & 11.16 & 12.93 & 4.70 & 20.00 & \multirow{3}{*}{.871} \\
\hline & & Female & 58 & 11.96 & 2.44 & 11.32 & 12.60 & 5.90 & 16.90 & \\
\hline & & Total & 100 & 12.00 & 2.60 & 11.48 & 12.51 & 4.70 & 20.00 & \\
\hline & \multirow{3}{*}{$\begin{array}{l}\text { Posterior } \\
\text { ( p) }\end{array}$} & Male & 42 & 16.28 & 3.28 & 15.25 & 17.30 & 11.20 & 24.30 & \multirow{3}{*}{.715} \\
\hline & & Female & 58 & 15.98 & 4.40 & 14.82 & 17.14 & 8.60 & 24.60 & \\
\hline & & Total & 100 & 16.11 & 3.95 & 15.32 & 16.89 & 8.60 & 24.60 & \\
\hline & \multirow{3}{*}{$\begin{array}{l}\text { Medial } \\
\text { (c) }\end{array}$} & Male & 42 & 13.04 & 2.68 & 12.21 & 13.88 & 7.77 & 20.60 & \multirow{3}{*}{.883} \\
\hline & & Female & 58 & 12.96 & 2.98 & 12.17 & 13.74 & 4.70 & 23.40 & \\
\hline & & Total & 100 & 12.99 & 2.85 & 12.43 & 13.56 & 4.70 & 23.40 & \\
\hline & \multirow{3}{*}{$\begin{array}{l}\text { lateral } \\
\text { (d) }\end{array}$} & Male & 42 & 15.63 & 3.95 & 14.40 & 16.86 & 8.20 & 25.70 & \multirow{3}{*}{.814} \\
\hline & & Female & 58 & 15.44 & 3.94 & 14.41 & 16.48 & 7.31 & 26.10 & \\
\hline & & Total & 100 & 15.52 & 3.92 & 14.74 & 16.30 & 7.31 & 26.10 & \\
\hline \multirow{12}{*}{ Right } & \multirow{3}{*}{$\begin{array}{l}\text { Anterior } \\
\text { (a) }\end{array}$} & Male & 42 & 12.04 & 2.91 & 11.13 & 12.95 & 7.00 & 16.70 & \multirow{3}{*}{.662} \\
\hline & & Female & 58 & 11.76 & 3.32 & 10.89 & 12.64 & 5.50 & 20.30 & \\
\hline & & Total & 100 & 11.88 & 3.14 & 11.25 & 12.50 & 5.50 & 20.30 & \\
\hline & \multirow{3}{*}{$\begin{array}{l}\text { Posterior } \\
\text { ( p) }\end{array}$} & Male & 42 & 16.74 & 4.20 & 15.43 & 18.05 & 10.50 & 26.50 & \multirow{3}{*}{.277} \\
\hline & & Female & 58 & 15.83 & 3.99 & 14.78 & 16.89 & 8.30 & 24.90 & \\
\hline & & Total & 100 & 16.21 & 4.09 & 15.40 & 17.03 & 8.30 & 26.50 & \\
\hline & \multirow{3}{*}{ medial (c) } & Male & 42 & 12.02 & 2.51 & 11.24 & 12.81 & 7.50 & 18.00 & \multirow{3}{*}{.314} \\
\hline & & Female & 58 & 11.53 & 2.30 & 10.93 & 12.14 & 5.90 & 17.70 & \\
\hline & & Total & 100 & 11.74 & 2.39 & 11.26 & 12.21 & 5.90 & 18.00 & \\
\hline & \multirow{3}{*}{ lateral (d) } & Male & 42 & 16.28 & 4.25 & 14.95 & 17.60 & 9.60 & 30.60 & \multirow{3}{*}{.110} \\
\hline & & Female & 58 & 15.04 & 3.39 & 14.15 & 15.93 & 7.34 & 22.20 & \\
\hline & & Total & 100 & 15.56 & 3.80 & 14.81 & 16.32 & 7.34 & 30.60 & \\
\hline
\end{tabular}

Table5: Descriptive Statistics of the Kidneys measurements at renal pelvis distributed according to gender and

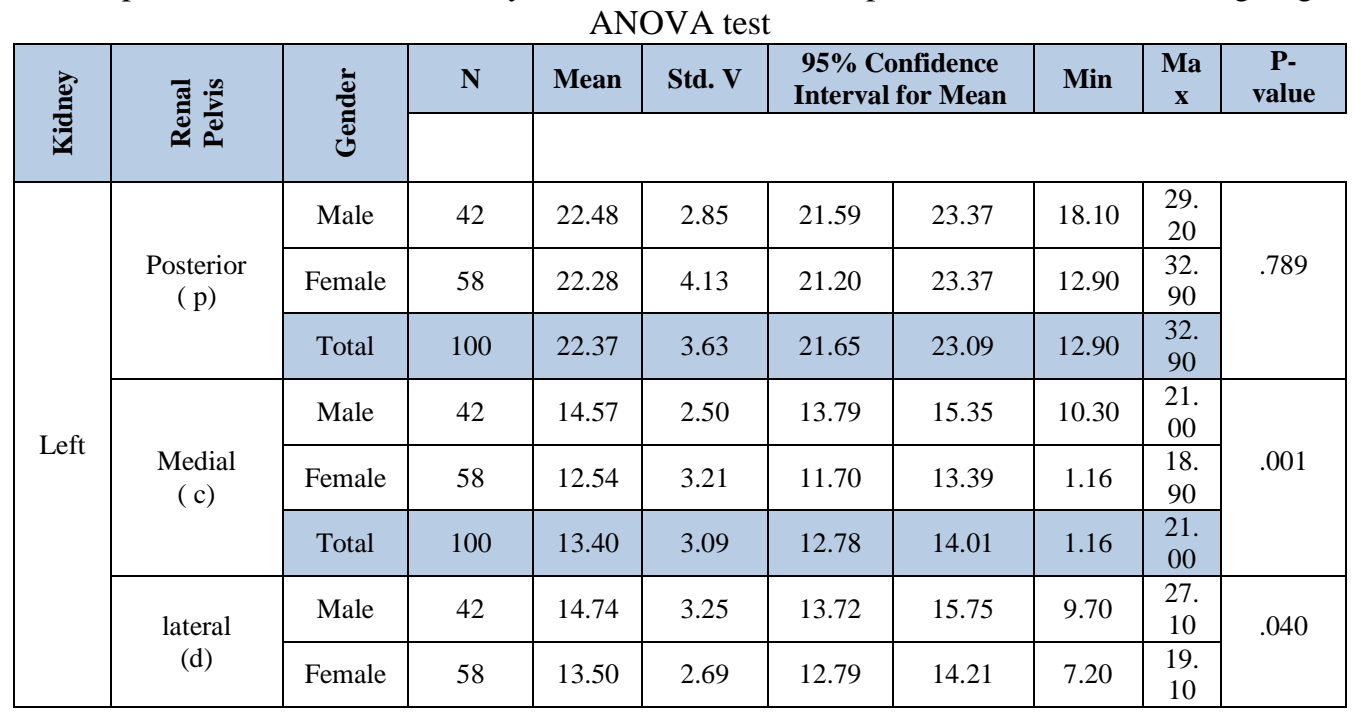


Normative Kidneys Measurements In Sudanese: A Morphometric CT Based Study

\begin{tabular}{|c|c|c|c|c|c|c|c|c|c|c|}
\hline & & Total & 100 & 14.02 & 2.99 & 13.42 & 14.61 & 7.20 & $\begin{array}{c}27 . \\
10\end{array}$ & \\
\hline \multirow{9}{*}{$\begin{array}{c}\text { Righ } \\
\mathrm{t}\end{array}$} & \multirow{3}{*}{$\begin{array}{l}\text { Posterior } \\
\text { (p) }\end{array}$} & Male & 42 & 23.22 & 3.48 & 22.14 & 24.31 & 17.10 & $\begin{array}{c}32 . \\
50\end{array}$ & \multirow{3}{*}{.026} \\
\hline & & Female & 57 & 21.58 & 3.65 & 20.61 & 22.55 & 14.50 & $\begin{array}{l}29 . \\
80\end{array}$ & \\
\hline & & Total & 99 & 22.28 & 3.65 & 21.55 & 23.01 & 14.50 & $\begin{array}{l}32 . \\
50\end{array}$ & \\
\hline & \multirow{3}{*}{$\begin{array}{l}\text { Medial } \\
\text { (c) }\end{array}$} & Male & 42 & 13.99 & 2.38 & 13.24 & 14.73 & 8.84 & $\begin{array}{l}18 . \\
80\end{array}$ & \multirow{3}{*}{.041} \\
\hline & & Female & 58 & 12.98 & 2.40 & 12.35 & 13.62 & 8.84 & $\begin{array}{l}20 . \\
40\end{array}$ & \\
\hline & & Total & 100 & 13.41 & 2.43 & 12.92 & 13.89 & 8.84 & $\begin{array}{l}20 . \\
40\end{array}$ & \\
\hline & \multirow{3}{*}{ lateral (d) } & Male & 42 & 15.21 & 3.61 & 14.08 & 16.34 & 10.10 & $\begin{array}{l}24 . \\
60\end{array}$ & \multirow{3}{*}{.040} \\
\hline & & Female & 58 & 13.70 & 3.55 & 12.76 & 14.63 & 6.62 & $\begin{array}{l}24 . \\
50\end{array}$ & \\
\hline & & Total & 100 & 14.33 & 3.64 & 13.61 & 15.05 & 6.62 & $\begin{array}{c}24 . \\
60\end{array}$ & \\
\hline
\end{tabular}

Table6: Descriptive Statistics of the Kidneys measurements at Lower Calyx distributed according to gender and ANOVA test

\begin{tabular}{|c|c|c|c|c|c|c|c|c|c|c|}
\hline 总 & $\begin{array}{l}\frac{x}{3} \\
\frac{3}{j} \\
\vdots \\
0\end{array}$ & 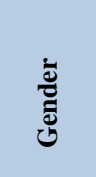 & $\mathbf{N}$ & $\underset{\mathbf{n}}{\mathrm{Mea}}$ & Std. V & \multicolumn{2}{|c|}{$\begin{array}{c}95 \% \\
\text { Confidence } \\
\text { Interval for } \\
\text { Mean } \\
\end{array}$} & Min & $\operatorname{Max}$ & p-Value \\
\hline \multirow{12}{*}{ Left } & \multirow{3}{*}{$\begin{array}{l}\text { Anterior } \\
\text { (a) }\end{array}$} & Male & 41 & $\begin{array}{c}10.8 \\
6\end{array}$ & 2.58 & 10.04 & $\begin{array}{c}11.6 \\
7\end{array}$ & 4.40 & 15.80 & \multirow{3}{*}{.923} \\
\hline & & Female & 58 & $\begin{array}{c}10.9 \\
0\end{array}$ & 2.37 & 10.28 & $\begin{array}{c}11.5 \\
3\end{array}$ & 6.40 & 17.30 & \\
\hline & & Total & 99 & $\begin{array}{c}10.8 \\
8 \\
\end{array}$ & 2.44 & 10.40 & $\begin{array}{c}11.3 \\
7 \\
\end{array}$ & 4.40 & 17.30 & \\
\hline & \multirow{3}{*}{$\begin{array}{l}\text { Posterior } \\
\quad(\mathrm{p})\end{array}$} & Male & 42 & $\begin{array}{c}19.5 \\
0 \\
\end{array}$ & 3.09 & 18.53 & $\begin{array}{c}20.4 \\
6 \\
\end{array}$ & 13.20 & 26.20 & \multirow{3}{*}{.152} \\
\hline & & Female & 58 & $\begin{array}{c}18.3 \\
7 \\
\end{array}$ & 4.28 & 17.25 & $\begin{array}{c}19.5 \\
0 \\
\end{array}$ & 8.80 & 28.80 & \\
\hline & & Total & 100 & $\begin{array}{c}18.8 \\
5\end{array}$ & 3.85 & 18.08 & $\begin{array}{c}19.6 \\
1\end{array}$ & 8.80 & 28.80 & \\
\hline & \multirow{3}{*}{$\begin{array}{l}\text { Medial } \\
\text { (c) }\end{array}$} & Male & 42 & $\begin{array}{c}12.7 \\
1 \\
\end{array}$ & 3.14 & 11.73 & $\begin{array}{c}13.6 \\
9 \\
\end{array}$ & 6.14 & 22.00 & \multirow{3}{*}{.479} \\
\hline & & Female & 57 & $\begin{array}{c}12.2 \\
6 \\
\end{array}$ & 3.06 & 11.45 & $\begin{array}{c}13.0 \\
8 \\
\end{array}$ & 6.50 & 23.40 & \\
\hline & & Total & 99 & $\begin{array}{c}12.4 \\
5 \\
\end{array}$ & 3.09 & 11.83 & $\begin{array}{c}13.0 \\
7 \\
\end{array}$ & 6.14 & 23.40 & \\
\hline & \multirow{3}{*}{$\begin{array}{l}\text { lateral } \\
\text { (d) }\end{array}$} & Male & 42 & $\begin{array}{c}12.5 \\
9\end{array}$ & 2.76 & 11.72 & $\begin{array}{c}13.4 \\
5\end{array}$ & 5.60 & 19.80 & \multirow{3}{*}{.357} \\
\hline & & Female & 58 & $\begin{array}{c}12.0 \\
8 \\
\end{array}$ & 2.65 & 11.38 & $\begin{array}{c}12.7 \\
8 \\
\end{array}$ & 6.78 & 22.00 & \\
\hline & & Total & 100 & $\begin{array}{c}12.2 \\
9\end{array}$ & 2.70 & 11.76 & $\begin{array}{c}12.8 \\
3\end{array}$ & 5.60 & 22.00 & \\
\hline \multirow{9}{*}{ Right } & \multirow{3}{*}{$\begin{array}{l}\text { Anterior } \\
\text { (a) }\end{array}$} & Male & 42 & $\begin{array}{c}32.2 \\
6 \\
\end{array}$ & 142.40 & 12.11 & $\begin{array}{c}76.6 \\
3 \\
\end{array}$ & 2.60 & 933.00 & \multirow{3}{*}{.240} \\
\hline & & Female & 58 & $\begin{array}{c}10.1 \\
7 \\
\end{array}$ & 3.04 & 9.37 & $\begin{array}{c}10.9 \\
7 \\
\end{array}$ & 4.00 & 19.60 & \\
\hline & & Total & 100 & $\begin{array}{c}19.4 \\
5 \\
\end{array}$ & 92.32 & 1.13 & $\begin{array}{c}37.7 \\
6 \\
\end{array}$ & 2.60 & 933.00 & \\
\hline & \multirow{3}{*}{$\begin{array}{l}\text { Posterior } \\
\text { (p) }\end{array}$} & Male & 42 & $\begin{array}{c}19.2 \\
0\end{array}$ & 3.51 & 18.11 & $\begin{array}{c}20.3 \\
0\end{array}$ & 11.90 & 27.10 & \multirow{3}{*}{.097} \\
\hline & & Female & 58 & $\begin{array}{c}17.8 \\
2 \\
\end{array}$ & 4.42 & 16.66 & $\begin{array}{c}18.9 \\
8 \\
\end{array}$ & 10.00 & 33.30 & \\
\hline & & Total & 100 & $\begin{array}{c}18.4 \\
0 \\
\end{array}$ & 4.10 & 17.59 & $\begin{array}{c}19.2 \\
2 \\
\end{array}$ & 10.00 & 33.30 & \\
\hline & \multirow{3}{*}{$\begin{array}{l}\text { Medial } \\
\text { (c) }\end{array}$} & Male & 42 & $\begin{array}{c}13.5 \\
8\end{array}$ & 2.91 & 12.68 & $\begin{array}{c}14.4 \\
9\end{array}$ & 8.36 & 22.30 & \multirow{3}{*}{.307} \\
\hline & & Female & 58 & $\begin{array}{c}12.8 \\
3 \\
\end{array}$ & 4.04 & 11.77 & $\begin{array}{c}13.9 \\
0 \\
\end{array}$ & 7.28 & 27.30 & \\
\hline & & Total & 100 & $\begin{array}{c}13.1 \\
5 \\
\end{array}$ & 3.61 & 12.43 & $\begin{array}{c}13.8 \\
7 \\
\end{array}$ & 7.28 & 27.30 & \\
\hline
\end{tabular}


Normative Kidneys Measurements In Sudanese: A Morphometric CT Based Study

\begin{tabular}{|c|c|c|c|c|c|c|c|c|c|c|}
\hline & \multirow{4}{*}{$\begin{array}{c}\text { lateral } \\
(\mathrm{d})\end{array}$} & Male & 42 & $\begin{array}{c}13.7 \\
3\end{array}$ & 2.95 & 12.81 & $\begin{array}{c}14.6 \\
5\end{array}$ & 9.80 & 22.00 & \\
\cline { 3 - 12 } & & Female & 58 & $\begin{array}{c}13.0 \\
7\end{array}$ & 3.87 & 12.05 & $\begin{array}{c}14.0 \\
8\end{array}$ & 7.68 & 25.70 & \multirow{2}{*}{.353} \\
\cline { 2 - 13 } & Total & 100 & $\begin{array}{c}13.3 \\
5\end{array}$ & 3.51 & 12.65 & $\begin{array}{c}14.0 \\
4\end{array}$ & 7.68 & 25.70 & \\
\hline
\end{tabular}

Table7: Descriptive Statistics of the Kidneys measurements distributed according to age and ANOVA test

\begin{tabular}{|c|c|c|c|c|c|c|c|c|c|c|}
\hline \multirow{2}{*}{ 矛 } & \multirow{2}{*}{$\frac{\varrho}{\frac{\pi}{\pi}}$} & \multirow{2}{*}{ 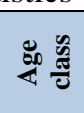 } & \multirow[t]{2}{*}{$\mathbf{N}$} & \multirow[t]{2}{*}{ Mean } & \multirow[t]{2}{*}{ Std. V } & \multicolumn{2}{|c|}{$\begin{array}{l}\text { 95\% Confidence } \\
\text { Interval for Mean }\end{array}$} & \multirow[t]{2}{*}{ Min } & \multirow[t]{2}{*}{ Max } & \multirow[t]{2}{*}{ P-value } \\
\hline & & & & & & & & & & \\
\hline \multirow{14}{*}{ Left } & \multirow{7}{*}{$\begin{array}{l}\text { Length } \\
\text { Of } \\
\text { Kidney }\end{array}$} & $10-19$ & 7 & 96.14 & 10.73 & 86.21 & 106.07 & 85.90 & 112.30 & \multirow[t]{7}{*}{.702} \\
\hline & & $20-29$ & 14 & 97.60 & 10.52 & 91.52 & 103.67 & 76.40 & 115.20 & \\
\hline & & $30-39$ & 19 & 93.17 & 10.77 & 87.98 & 98.36 & 65.90 & 114.50 & \\
\hline & & $40-49$ & 23 & 98.43 & 11.29 & 93.55 & 103.31 & 82.20 & 121.20 & \\
\hline & & $50-59$ & 18 & 94.19 & 15.70 & 86.38 & 102.00 & 65.30 & 123.40 & \\
\hline & & $60-70$ & 19 & 94.95 & 8.36 & 90.92 & 98.98 & 81.30 & 114.80 & \\
\hline & & Total & 100 & 95.73 & 11.40 & 93.47 & 97.99 & 65.30 & 123.40 & \\
\hline & \multirow{7}{*}{$\begin{array}{l}\text { Width } \\
\text { Of } \\
\text { Kidney }\end{array}$} & $10-19$ & 7 & 40.58 & 4.98 & 35.97 & 45.19 & 34.10 & 46.00 & \multirow[t]{7}{*}{.315} \\
\hline & & $20-29$ & 14 & 40.74 & 5.04 & 37.82 & 43.65 & 33.40 & 52.00 & \\
\hline & & $30-39$ & 19 & 41.73 & 3.93 & 39.84 & 43.63 & 33.20 & 47.10 & \\
\hline & & 40-49 & 23 & 43.25 & 3.65 & 41.66 & 44.83 & 37.50 & 54.00 & \\
\hline & & 50-59 & 18 & 44.38 & 5.17 & 41.81 & 46.96 & 35.90 & 57.60 & \\
\hline & & $60-70$ & 19 & 42.65 & 7.30 & 39.13 & 46.17 & 31.50 & 55.30 & \\
\hline & & Total & 100 & 42.51 & 5.15 & 41.49 & 43.53 & 31.50 & 57.60 & \\
\hline \multirow{14}{*}{ Right } & \multirow{7}{*}{$\begin{array}{l}\text { Length } \\
\text { Of } \\
\text { Kidney }\end{array}$} & 10-19 & 7 & 96.88 & 13.57 & 84.33 & 109.43 & 79.60 & 116.50 & \multirow[t]{7}{*}{0.255} \\
\hline & & $20-29$ & 14 & 94.50 & 10.80 & 88.27 & 100.74 & 75.70 & 111.70 & \\
\hline & & $30-39$ & 18 & 94.35 & 8.09 & 90.32 & 98.38 & 79.70 & 110.00 & \\
\hline & & 40-49 & 23 & 99.95 & 8.37 & 96.32 & 103.57 & 83.10 & 118.40 & \\
\hline & & $50-59$ & 19 & 99.04 & 9.64 & 94.39 & 103.69 & 75.30 & 121.90 & \\
\hline & & 60-70 & 19 & 94.46 & 9.41 & 89.92 & 99.00 & 76.30 & 113.40 & \\
\hline & & Total & 100 & 96.75 & 9.61 & 94.84 & 98.66 & 75.30 & 121.90 & \\
\hline & \multirow{7}{*}{$\begin{array}{l}\text { Width } \\
\text { Of } \\
\text { Kidney }\end{array}$} & 10-19 & 7 & 39.48 & 4.72 & 35.11 & 43.85 & 34.20 & 47.70 & \multirow[t]{7}{*}{0.254} \\
\hline & & $20-29$ & 14 & 39.01 & 4.82 & 36.22 & 41.79 & 30.00 & 45.80 & \\
\hline & & $30-39$ & 18 & 40.91 & 3.66 & 39.08 & 42.73 & 33.80 & 48.10 & \\
\hline & & $40-49$ & 23 & 40.30 & 4.51 & 38.35 & 42.26 & 31.60 & 49.40 & \\
\hline & & $50-59$ & 19 & 43.10 & 5.97 & 40.22 & 45.98 & 33.50 & 51.90 & \\
\hline & & $60-70$ & 19 & 41.87 & 6.61 & 38.68 & 45.06 & 31.70 & 55.90 & \\
\hline & & Total & 100 & 41.00 & 5.24 & 39.96 & 42.04 & 30.00 & 55.90 & \\
\hline
\end{tabular}

Table8: Descriptive Statistics of the Kidneys cortex and medulla measurements and CT (HU) distributed according to age and ANOVA test

\begin{tabular}{|c|c|c|c|c|c|c|c|c|c|c|}
\hline \multirow{2}{*}{ 冚 } & \multirow{2}{*}{ 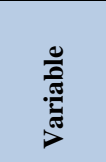 } & \multirow{2}{*}{ 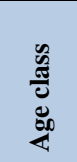 } & $\mathbf{N}$ & Mean & Std. V & $\begin{array}{r}95 \% \mathrm{C} \\
\text { Inte } \\
\end{array}$ & $\begin{array}{l}\text { idence } \\
\text { for } \\
\end{array}$ & Min & Max & \multirow[t]{2}{*}{ P-value } \\
\hline & & & & & & & & & & \\
\hline \multirow{14}{*}{ Left } & \multirow{7}{*}{$\begin{array}{l}\text { Cortex } \\
\text { (axial) }\end{array}$} & $10-19$ & 7 & 5.16 & .53 & 4.66 & 5.65 & 4.22 & 6.00 & \multirow[t]{7}{*}{.400} \\
\hline & & $20-29$ & 14 & 5.69 & .73 & 5.26 & 6.11 & 4.45 & 6.70 & \\
\hline & & $30-39$ & 19 & 5.19 & .88 & 4.76 & 5.61 & 3.72 & 6.85 & \\
\hline & & $40-49$ & 23 & 5.12 & .83 & 4.76 & 5.48 & 3.20 & 6.60 & \\
\hline & & $50-59$ & 18 & 5.15 & .81 & 4.75 & 5.56 & 3.40 & 6.00 & \\
\hline & & $60-70$ & 18 & 5.36 & .91 & 4.90 & 5.81 & 3.10 & 6.10 & \\
\hline & & Total & 99 & 5.26 & .82 & 5.10 & 5.43 & 3.10 & 6.85 & \\
\hline & \multirow{7}{*}{$\begin{array}{l}\text { Medulla } \\
\text { (axial) }\end{array}$} & 10-19 & 7 & 10.11 & 1.96 & 8.28 & 11.93 & 7.40 & 12.08 & \multirow[t]{7}{*}{.318} \\
\hline & & $20-29$ & 14 & 11.10 & 1.53 & 10.21 & 11.99 & 8.10 & 13.60 & \\
\hline & & $30-39$ & 19 & 11.02 & 1.58 & 10.25 & 11.78 & 7.10 & 13.10 & \\
\hline & & $40-49$ & 23 & 11.07 & 1.53 & 10.40 & 11.73 & 6.80 & 13.90 & \\
\hline & & $50-59$ & 18 & 11.59 & 1.65 & 10.77 & 12.42 & 9.00 & 15.20 & \\
\hline & & $60-70$ & 19 & 11.68 & 1.91 & 10.76 & 12.61 & 9.34 & 17.60 & \\
\hline & & Total & 100 & 11.21 & 1.68 & 10.87 & 11.54 & 6.80 & 17.60 & \\
\hline \multirow{3}{*}{ Right } & \multirow{3}{*}{$\begin{array}{l}\text { Cortex } \\
\text { (axial) }\end{array}$} & $10-19$ & 7 & 5.23 & .82 & 4.46 & 5.99 & 4.09 & 6.70 & \multirow[t]{3}{*}{.873} \\
\hline & & $20-29$ & 14 & 5.45 & .63 & 5.09 & 5.82 & 4.40 & 6.50 & \\
\hline & & $30-39$ & 18 & 5.11 & .84 & 4.69 & 5.53 & 3.67 & 6.60 & \\
\hline
\end{tabular}


Normative Kidneys Measurements In Sudanese: A Morphometric CT Based Study

\begin{tabular}{|c|c|c|c|c|c|c|c|c|c|}
\hline & $40-49$ & 23 & 5.15 & .81 & 4.80 & 5.51 & 3.31 & 6.90 & \\
\hline & $50-59$ & 18 & 5.12 & .82 & 4.71 & 5.53 & 3.30 & 6.30 & \\
\hline & $60-70$ & 19 & 5.26 & 1.00 & 4.78 & 5.75 & 3.20 & 6.50 & \\
\hline & Total & 99 & 5.21 & .82 & 5.04 & 5.37 & 3.20 & 6.90 & \\
\hline \multirow{8}{*}{$\begin{array}{l}\text { Medulla } \\
\text { (axial) }\end{array}$} & $10-19$ & 7 & 9.38 & 2.09 & 7.44 & 11.31 & 5.40 & 12.07 & \multirow[t]{8}{*}{.109} \\
\hline & $20-29$ & 14 & 10.75 & 1.49 & 9.89 & 11.62 & 8.30 & 13.30 & \\
\hline & $30-39$ & 18 & 10.48 & 1.91 & 9.53 & 11.43 & 7.40 & 14.00 & \\
\hline & $40-49$ & 23 & 10.64 & 1.72 & 9.90 & 11.39 & 6.50 & 13.70 & \\
\hline & $50-59$ & 18 & 11.26 & 1.891 & 10.32 & 12.20 & 6.80 & 14.10 & \\
\hline & $60-70$ & 19 & 11.53 & 1.82 & 10.65 & 12.41 & 7.80 & 14.80 & \\
\hline & Total & 99 & 10.82 & 1.84 & 10.45 & 11.19 & 5.40 & 14.80 & \\
\hline & Total & 99 & 37.23 & 4.63 & 36.30 & 38.15 & $\begin{array}{c}29.0 \\
0\end{array}$ & 49.00 & \\
\hline
\end{tabular}

Table9: Descriptive Statistics of both Kidneys upper calyx measurements at different sites distributed according

\begin{tabular}{|c|c|c|c|c|c|c|c|c|c|c|}
\hline 冚 & 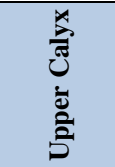 & 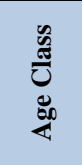 & $\mathbf{N}$ & Mean & Std. V & \multicolumn{2}{|c|}{$\begin{array}{l}\text { 95\% Confidence } \\
\text { Interval for } \\
\text { Mean }\end{array}$} & Min & Max & P-Value \\
\hline \multirow{28}{*}{ Left } & \multirow{7}{*}{$\begin{array}{l}\text { Anterior } \\
\text { (a) }\end{array}$} & $10-19$ & 7 & 12.25 & 1.37 & 10.98 & 13.53 & 11.00 & 14.70 & \multirow{7}{*}{.959} \\
\hline & & $20-29$ & 14 & 11.60 & 3.12 & 9.80 & 13.41 & 4.70 & 16.70 & \\
\hline & & $30-39$ & 19 & 11.87 & 2.73 & 10.55 & 13.18 & 5.90 & 16.60 & \\
\hline & & $40-49$ & 23 & 11.85 & 2.47 & 10.78 & 12.92 & 8.20 & 16.90 & \\
\hline & & $50-59$ & 18 & 12.46 & 2.96 & 10.98 & 13.93 & 9.30 & 20.00 & \\
\hline & & $60-70$ & 19 & 12.06 & 2.43 & 10.89 & 13.23 & 7.90 & 16.80 & \\
\hline & & Total & 100 & 12.00 & 2.60 & 11.48 & 12.51 & 4.70 & 20.00 & \\
\hline & \multirow{7}{*}{$\begin{array}{l}\text { posterior } \\
\text { (p) }\end{array}$} & $10-19$ & 7 & 16.85 & 5.61 & 11.66 & 22.05 & 9.20 & 22.90 & \multirow[t]{7}{*}{.689} \\
\hline & & $20-29$ & 14 & 16.57 & 3.39 & 14.60 & 18.53 & 11.40 & 22.50 & \\
\hline & & $30-39$ & 19 & 14.88 & 3.58 & 13.15 & 16.61 & 8.60 & 24.60 & \\
\hline & & $40-49$ & 23 & 16.23 & 4.43 & 14.31 & 18.15 & 9.20 & 23.20 & \\
\hline & & $50-59$ & 18 & 16.91 & 3.71 & 15.06 & 18.75 & 11.40 & 24.30 & \\
\hline & & $60-70$ & 19 & 15.81 & 3.80 & 13.97 & 17.64 & 9.30 & 22.70 & \\
\hline & & Total & 100 & 16.11 & 3.95 & 15.32 & 16.89 & 8.60 & 24.60 & \\
\hline & \multirow{7}{*}{$\begin{array}{l}\text { medial } \\
\text { (c) }\end{array}$} & $10-19$ & 7 & 13.36 & 5.20 & 8.54 & 18.18 & 7.77 & 23.40 & \multirow[t]{7}{*}{.640} \\
\hline & & $20-29$ & 14 & 12.94 & 2.53 & 11.47 & 14.40 & 10.00 & 18.00 & \\
\hline & & $30-39$ & 19 & 12.05 & 3.15 & 10.53 & 13.57 & 4.70 & 17.70 & \\
\hline & & $40-49$ & 23 & 12.86 & 1.91 & 12.03 & 13.69 & 9.50 & 16.20 & \\
\hline & & $50-59$ & 18 & 13.46 & 2.26 & 12.33 & 14.58 & 8.50 & 16.70 & \\
\hline & & $60-70$ & 19 & 13.56 & 3.19 & 12.02 & 15.10 & 8.00 & 20.60 & \\
\hline & & Total & 100 & 12.99 & 2.85 & 12.43 & 13.56 & 4.70 & 23.40 & \\
\hline & \multirow{7}{*}{$\begin{array}{l}\text { Lateral } \\
\text { (d) }\end{array}$} & 10-19 & 7 & 14.90 & 4.96 & 10.30 & 19.49 & 10.40 & 22.40 & \multirow[t]{7}{*}{.930} \\
\hline & & $20-29$ & 14 & 14.77 & 3.72 & 12.62 & 16.92 & 9.70 & 21.10 & \\
\hline & & 30-39 & 19 & 15.22 & 4.31 & 13.14 & 17.30 & 7.31 & 25.70 & \\
\hline & & $40-49$ & 23 & 16.06 & 4.02 & 14.31 & 17.80 & 9.58 & 26.10 & \\
\hline & & $50-59$ & 18 & 15.60 & 3.51 & 13.85 & 17.34 & 8.20 & 21.60 & \\
\hline & & $60-70$ & 19 & 15.88 & 3.90 & 14.00 & 17.77 & 9.10 & 20.70 & \\
\hline & & Total & 100 & 15.52 & 3.92 & 14.74 & 16.30 & 7.31 & 26.10 & \\
\hline \multirow{13}{*}{ Right } & \multirow{7}{*}{$\begin{array}{l}\text { Anterior } \\
\text { (a) }\end{array}$} & $10-19$ & 7 & 13.37 & 3.46 & 10.16 & 16.58 & 9.20 & 19.50 & \multirow[t]{7}{*}{.168} \\
\hline & & $20-29$ & 14 & 11.67 & 3.73 & 9.52 & 13.83 & 5.50 & 17.40 & \\
\hline & & $30-39$ & 18 & 12.73 & 3.30 & 11.08 & 14.37 & 7.36 & 20.30 & \\
\hline & & $40-49$ & 23 & 12.29 & 2.92 & 11.02 & 13.56 & 7.50 & 18.40 & \\
\hline & & $50-59$ & 19 & 10.37 & 2.94 & 8.95 & 11.79 & 6.27 & 18.20 & \\
\hline & & $60-70$ & 19 & 11.69 & 2.58 & 10.44 & 12.94 & 7.33 & 16.50 & \\
\hline & & Total & 100 & 11.88 & 3.14 & 11.25 & 12.50 & 5.50 & 20.30 & \\
\hline & \multirow{6}{*}{$\begin{array}{l}\text { posterior } \\
\text { (p) }\end{array}$} & $10-19$ & 7 & 18.10 & 2.07 & 16.18 & 20.01 & 14.70 & 20.70 & .842 \\
\hline & & $20-29$ & 14 & 16.27 & 4.93 & 13.42 & 19.12 & 8.30 & 23.70 & \\
\hline & & $30-39$ & 18 & 16.38 & 3.53 & 14.62 & 18.13 & 9.30 & 24.90 & \\
\hline & & $40-49$ & 23 & 15.92 & 4.30 & 14.06 & 17.78 & 9.07 & 23.20 & \\
\hline & & $50-59$ & 19 & 16.3 & 3.76 & 14.51 & 18.14 & 11.90 & 25.30 & \\
\hline & & $60-70$ & 19 & 15.57 & 4.74 & 13.29 & 17.86 & 10.50 & 26.50 & \\
\hline
\end{tabular}


Normative Kidneys Measurements In Sudanese: A Morphometric CT Based Study

\begin{tabular}{|c|c|c|c|c|c|c|c|c|c|}
\hline & Total & 100 & 16.21 & 4.09 & 15.40 & 17.03 & 8.30 & 26.50 & \\
\hline \multirow{7}{*}{$\begin{array}{c}\text { Medial } \\
\text { (c) }\end{array}$} & 10-19 & 7 & 11.33 & 2.65 & 8.87 & 13.78 & 9.01 & 16.00 & \multirow[t]{7}{*}{.715} \\
\hline & $20-29$ & 14 & 11.42 & 2.10 & 10.21 & 12.64 & 8.00 & 14.50 & \\
\hline & 30-39 & 18 & 11.22 & 2.39 & 10.02 & 12.41 & 5.90 & 17.60 & \\
\hline & $40-49$ & 23 & 11.82 & 2.53 & 10.72 & 12.91 & 8.10 & 17.80 & \\
\hline & 50-59 & 19 & 11.83 & 2.10 & 10.81 & 12.84 & 7.50 & 18.00 & \\
\hline & $60-70$ & 19 & 12.44 & 2.71 & 11.13 & 13.75 & 8.10 & 17.70 & \\
\hline & Total & 100 & 11.74 & 2.39 & 11.26 & 12.21 & 5.90 & 18.00 & \\
\hline \multirow{7}{*}{$\begin{array}{l}\text { lateral } \\
\text { (d) }\end{array}$} & $10-19$ & 7 & 14.64 & 3.38 & 11.51 & 17.76 & 11.20 & 20.40 & \multirow[t]{7}{*}{.440} \\
\hline & $20-29$ & 14 & 14.02 & 3.24 & 12.14 & 15.89 & 9.60 & 19.70 & \\
\hline & $30-39$ & 18 & 15.80 & 4.33 & 13.65 & 17.95 & 7.34 & 23.90 & \\
\hline & $40-49$ & 23 & 16.70 & 3.59 & 15.15 & 18.25 & 11.20 & 25.00 & \\
\hline & $50-59$ & 19 & 15.45 & 3.09 & 13.96 & 16.94 & 10.40 & 21.70 & \\
\hline & $60-70$ & 19 & 15.54 & 4.62 & 13.32 & 17.77 & 10.00 & 30.60 & \\
\hline & Total & 100 & 15.56 & 3.80 & 14.81 & 16.32 & 7.34 & 30.60 & \\
\hline
\end{tabular}

Table10: Descriptive Statistics of both Kidneys renal pelvis measurements at different sites distributed

\begin{tabular}{|c|c|c|c|c|c|c|c|c|c|c|}
\hline 冚 & 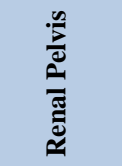 & 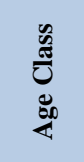 & $\mathbf{N}$ & Mean & Std. V & \multicolumn{2}{|c|}{$\begin{array}{l}95 \% \text { Confidence } \\
\text { Interval For Mean }\end{array}$} & Min & Max & P-Value \\
\hline \multirow{21}{*}{ Left } & \multirow{7}{*}{$\begin{array}{l}\text { Posterior } \\
\text { (p) }\end{array}$} & $10-19$ & 7 & 20.57 & 2.58 & 18.18 & 22.95 & 17.80 & 26.10 & \multirow{7}{*}{.166} \\
\hline & & $20-29$ & 14 & 23.66 & 4.30 & 21.17 & 26.15 & 19.90 & 32.90 & \\
\hline & & $30-39$ & 19 & 21.08 & 3.52 & 19.39 & 22.78 & 13.60 & 26.30 & \\
\hline & & $40-49$ & 23 & 23.41 & 2.53 & 22.31 & 24.51 & 19.20 & 29.00 & \\
\hline & & $50-59$ & 18 & 22.21 & 4.34 & 20.04 & 24.37 & 13.50 & 31.50 & \\
\hline & & $60-70$ & 19 & 22.25 & 3.64 & 20.50 & 24.01 & 12.90 & 27.10 & \\
\hline & & Total & 100 & 22.37 & 3.63 & 21.65 & 23.09 & 12.90 & 32.90 & \\
\hline & \multirow{7}{*}{$\begin{array}{l}\text { Medial } \\
\text { (c) }\end{array}$} & $10-19$ & 7 & 12.28 & 3.21 & 9.31 & 15.26 & 10.30 & 18.70 & \multirow{7}{*}{.264} \\
\hline & & $20-29$ & 14 & 12.27 & 3.53 & 10.22 & 14.31 & 6.80 & 18.90 & \\
\hline & & $30-39$ & 19 & 13.16 & 2.59 & 11.91 & 14.41 & 9.50 & 19.80 & \\
\hline & & $40-49$ & 23 & 13.14 & 3.44 & 11.65 & 14.63 & 1.16 & 20.70 & \\
\hline & & $50-59$ & 18 & 14.12 & 2.24 & 13.01 & 15.24 & 8.90 & 17.90 & \\
\hline & & $60-70$ & 19 & 14.50 & 3.26 & 12.93 & 16.07 & 6.60 & 21.00 & \\
\hline & & Total & 100 & 13.40 & 3.09 & 12.78 & 14.01 & 1.16 & 21.00 & \\
\hline & \multirow{7}{*}{$\begin{array}{l}\text { lateral } \\
\text { (d) }\end{array}$} & $10-19$ & 7 & 13.70 & 2.25 & 11.61 & 15.78 & 10.50 & 15.60 & \multirow[t]{7}{*}{.006} \\
\hline & & $20-29$ & 14 & 12.13 & 3.15 & 10.30 & 13.95 & 7.20 & 19.10 & \\
\hline & & $30-39$ & 19 & 14.78 & 3.15 & 13.26 & 16.31 & 7.60 & 20.00 & \\
\hline & & $40-49$ & 23 & 13.26 & 1.98 & 12.41 & 14.12 & 10.10 & 17.70 & \\
\hline & & $50-59$ & 18 & 13.85 & 2.04 & 12.83 & 14.87 & 9.67 & 16.60 & \\
\hline & & $60-70$ & 19 & 15.84 & 3.74 & 14.03 & 17.64 & 11.50 & 27.10 & \\
\hline & & Total & 100 & 14.02 & 2.99 & 13.42 & 14.61 & 7.20 & 27.10 & \\
\hline \multirow{21}{*}{ Right } & \multirow{7}{*}{$\begin{array}{l}\text { Posterior } \\
\text { (p) }\end{array}$} & 10-19 & 7 & 22.80 & 3.35 & 19.70 & 25.89 & 20.20 & 28.40 & \multirow[t]{7}{*}{.841} \\
\hline & & $20-29$ & 14 & 22.32 & 3.57 & 20.25 & 24.38 & 19.00 & 29.80 & \\
\hline & & 30-39 & 18 & 22.20 & 3.35 & 20.53 & 23.87 & 15.50 & 28.80 & \\
\hline & & $40-49$ & 23 & 21.74 & 2.67 & 20.58 & 22.89 & 16.00 & 25.60 & \\
\hline & & 50-59 & 19 & 21.87 & 4.71 & 19.60 & 24.14 & 14.50 & 32.50 & \\
\hline & & $60-70$ & 18 & 23.23 & 4.21 & 21.14 & 25.33 & 14.80 & 28.90 & \\
\hline & & Total & 99 & 22.28 & 3.65 & 21.55 & 23.01 & 14.50 & 32.50 & \\
\hline & \multirow{7}{*}{$\begin{array}{l}\text { Medial } \\
\text { (c) }\end{array}$} & $10-19$ & 7 & 11.86 & 2.33 & 9.71 & 14.02 & 8.84 & 14.70 & \multirow[t]{7}{*}{.445} \\
\hline & & $20-29$ & 14 & 12.93 & 2.27 & 11.62 & 14.25 & 9.30 & 18.50 & \\
\hline & & $30-39$ & 18 & 13.37 & 2.43 & 12.16 & 14.58 & 9.43 & 17.30 & \\
\hline & & $40-49$ & 23 & 13.94 & 2.44 & 12.89 & 15.00 & 10.30 & 18.80 & \\
\hline & & $50-59$ & 19 & 13.74 & 3.03 & 12.27 & 15.20 & 9.00 & 20.40 & \\
\hline & & $60-70$ & 19 & 13.37 & 1.83 & 12.49 & 14.26 & 10.20 & 17.40 & \\
\hline & & Total & 100 & 13.41 & 2.43 & 12.92 & 13.89 & 8.84 & 20.40 & \\
\hline & \multirow{7}{*}{$\begin{array}{l}\text { Lateral } \\
\text { (d) }\end{array}$} & $10-19$ & 7 & 14.14 & 3.06 & 11.30 & 16.97 & 11.00 & 20.00 & \multirow[t]{7}{*}{.416} \\
\hline & & $20-29$ & 14 & 13.30 & 3.27 & 11.41 & 15.20 & 10.40 & 21.70 & \\
\hline & & $30-39$ & 18 & 15.59 & 3.51 & 13.84 & 17.34 & 11.30 & 24.60 & \\
\hline & & $40-49$ & 23 & 13.46 & 4.00 & 11.72 & 15.19 & 6.62 & 24.50 & \\
\hline & & $50-59$ & 19 & 14.51 & 4.02 & 12.58 & 16.45 & 9.76 & 21.80 & \\
\hline & & $60-70$ & 19 & 14.85 & 3.29 & 13.26 & 16.44 & 9.15 & 22.20 & \\
\hline & & Total & 100 & 14.33 & 3.64 & 13.61 & 15.05 & 6.62 & 24.60 & \\
\hline
\end{tabular}


Normative Kidneys Measurements In Sudanese: A Morphometric CT Based Study

Table11: Descriptive Statistics of both Kidneys lower calyex measurements at different sites distributed according to age and ANOVA test

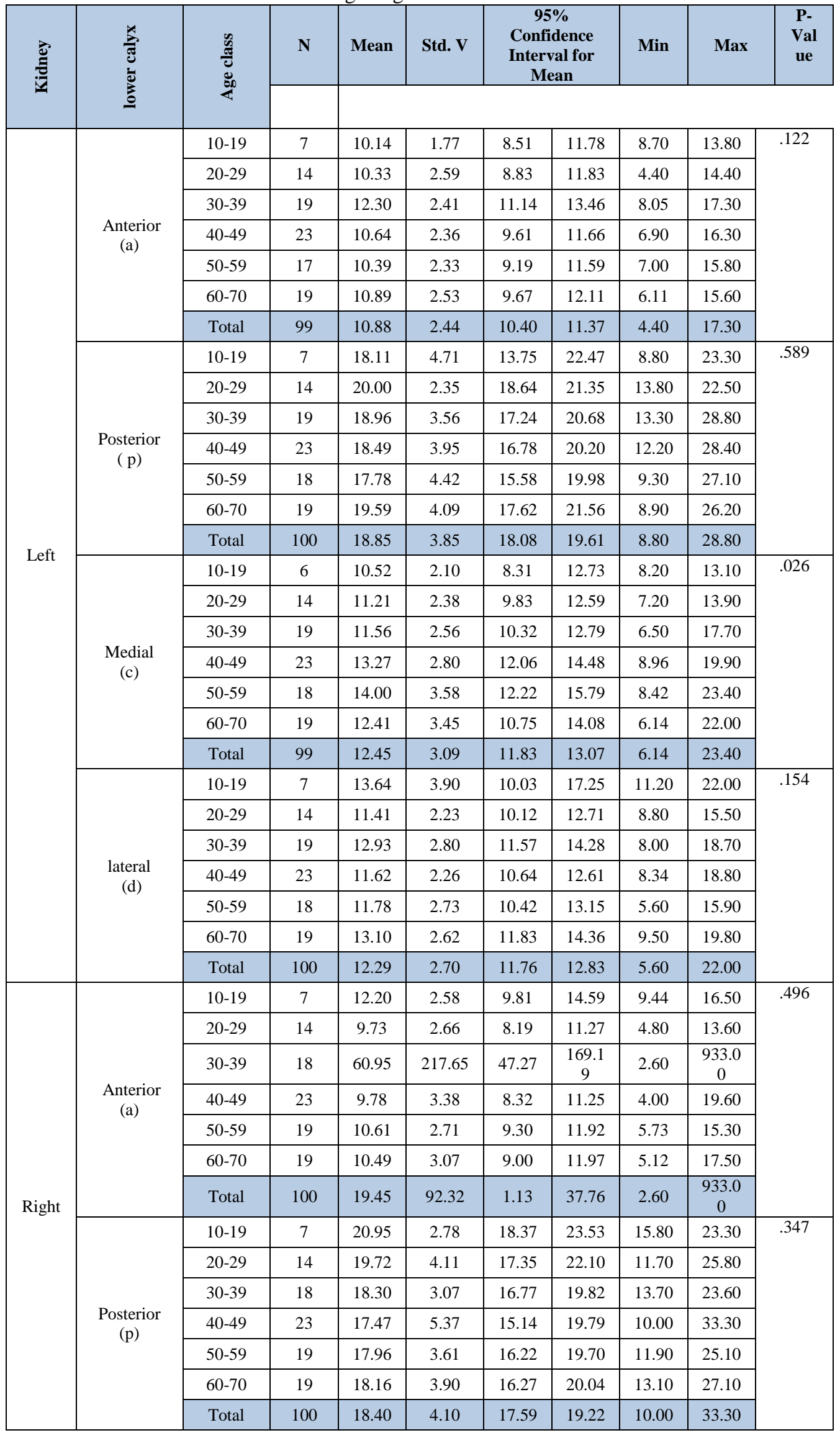


Normative Kidneys Measurements In Sudanese: A Morphometric CT Based Study

\begin{tabular}{|c|c|c|c|c|c|c|c|c|c|}
\hline \multirow{7}{*}{$\begin{array}{l}\text { Medial } \\
\text { (c) }\end{array}$} & $10-19$ & 7 & 11.82 & 3.62 & 8.46 & 15.17 & 7.90 & 17.60 & \multirow[t]{7}{*}{.292} \\
\hline & 20-29 & 14 & 12.47 & 3.23 & 10.60 & 14.33 & 8.21 & 18.70 & \\
\hline & $30-39$ & 18 & 14.93 & 4.94 & 12.47 & 17.38 & 7.28 & 27.30 & \\
\hline & 40-49 & 23 & 13.17 & 3.77 & 11.54 & 14.80 & 8.10 & 19.80 & \\
\hline & 50-59 & 19 & 12.86 & 3.06 & 11.39 & 14.34 & 8.26 & 17.60 & \\
\hline & $60-70$ & 19 & 12.72 & 2.38 & 11.57 & 13.87 & 8.87 & 17.60 & \\
\hline & Total & 100 & 13.15 & 3.61 & 12.43 & 13.87 & 7.28 & 27.30 & \\
\hline \multirow{7}{*}{$\begin{array}{l}\text { Lateral } \\
\text { (d) }\end{array}$} & $10-19$ & 7 & 14.78 & 2.48 & 12.48 & 17.08 & 11.70 & 18.90 & \multirow[t]{7}{*}{.556} \\
\hline & $20-29$ & 14 & 13.20 & 2.31 & 11.87 & 14.54 & 10.40 & 18.70 & \\
\hline & 30-39 & 18 & 14.12 & 4.41 & 11.92 & 16.32 & 7.68 & 25.70 & \\
\hline & $40-49$ & 23 & 13.33 & 4.46 & 11.39 & 15.26 & 7.97 & 24.40 & \\
\hline & $50-59$ & 19 & 12.22 & 2.70 & 10.92 & 13.53 & 7.74 & 17.20 & \\
\hline & $60-70$ & 19 & 13.34 & 3.01 & 11.88 & 14.79 & 8.50 & 22.00 & \\
\hline & Total & 100 & 13.35 & 3.51 & 12.65 & 14.04 & 7.68 & 25.70 & \\
\hline
\end{tabular}

\section{Discussion}

Normal renal measurements are an important issue in studying renal physiology and its disorders. It is also important in making a primary diagnosis as well as during the follow-up of patients with renal diseases, in order to monitor the diseases' development. [18]

Data that were obtained for both left and right kidney from all the participants were renal length, width and CT number as well as renal character in the upper pole, calyces and lower pole as well as renal pelvis. All measurements were taken in mm. When comparing the right and left kidneys the study showed that there is no significant difference between the two kidneys length where there were significant difference in kidneys width at $\mathrm{p} \leq 0.041$, left kidney is greater than the right by $1.5 \mathrm{~cm}$ (table 1 ).

Our research agreed with previous studies done that left kidney is larger than the right [19-21] Table (1) shows the measurements of the kidneys as mean and standard deviation values. Both kidneys measurements at the upper calyx, renal pelvis, Lower calyx measured at all directions showed that the medial upper calyces for the left kidney measured in axial direction is larger significantly than the right kidney at $\mathrm{p}=0.001$ as well as the lateral (d) of the lower calyx differs significantly between the two kidneys at $\mathrm{p}=0.018$. Table (2). The likely justification is, due to the size of spleen which is smaller than the liver, thus the left kidney has more space for its growth. Another possible cause is that because of the left renal artery is shorter and straighter than the right one; this causes increased blood flow in the left artery which may result in relatively increased in measurements $[22,23]$

The present data show that the kidney character including kidney length and width, cortex and medulla width were independent of gender; similar findings in other population have been mentioned. [24, 25] From the data obtained, we can also conclude that renal measurements in female population are relatively smaller compared to male population. However, the differences in renal measurements for upper and lower calyxes found in this study are quite minimal and not significant

Except for the measurement of the renal pelvis measurement were significantly differ between both genders for medial , lateral measurements in both right and left kidneys at $\mathrm{p}=0.001,0.040$ and $0.041,0.040$ respectively. The posterior measurement for the right kidney differed significantly between both genders at $\mathrm{p}=0.026$. In all cases the males have greater measurements than females regarding renal pelvis measurements. Tables $(3,4,5,6)$

The gender are not a predictor for changes in the renal measurements except for the medial and lateral renal pelvis segments for both right and left kidneys at $(P=0.041,0.040$ and $0.001,0.040)$. The age also is not a predictor for renal measurement except the renal pelvis lateral segment of the left kidney as well as lower calyx medial segment at $\mathrm{p}=0.006$ and0.026.Tables (7-11).A new chart for renal character and measurement were established according to different age classes from 10 years to 70 years and for both gendered s for Sudanese population.

Our study showed that kidney length was $96.75 \mathrm{~mm}$ for the right, and $95.73 \mathrm{~mm}$ for the left. The right kidneys width was $41.00 \mathrm{~mm}$, less than the left $42.51 \mathrm{~mm}$ at $(\mathrm{p}=0.041$ ) when compared to the study done by Emamian et al.[26] based on Denmark population which consist of different ranged of age had shown that the renal lengths were $11.2 \mathrm{~cm}$ on the left side and $10.9 \mathrm{~cm}$ on the right side. The mean renal length for Denmark differs from Sudanese. Another study from Mexican population by J. Oyuela- Carrasco et al. [27] had reported the renal length in Mexican adults differs as well as the gender has an impact in the kidneys measurements. 
What we can say here is that all the differences in the previous studies were related to ethnic groups pointed that the results of this current study differ from other populations. Danish population left kidney length was found to be $11.2 \mathrm{~cm}$ and the right kidney was $10.9 \mathrm{~cm}$, [26] In Mexicans; kidney length was $10.5 \mathrm{~cm}$ for the left and $10.4 \mathrm{~cm}$ for the right. [27] Nigerian population has kidney length of $10.6 \mathrm{~cm}$ for the left and $10.3 \mathrm{~cm}$ for the right. [28] The Northwest Indian populations' measurements were $10.0 \mathrm{~cm}$ for the left kidney length and $9.9 \mathrm{~cm}$ for the right kidney [.29] Japanese have left kidney length of $11.5 \mathrm{~cm}$,right of $11.3 \mathrm{~cm}$ and width of 5.7 $\mathrm{cm}, 5.5 \mathrm{~cm}$ for left and right in respectively [30] Caucasians population have $12 \mathrm{~cm}$ kidneys length and $6 \mathrm{~cm}$ kidneys width as mentioned by Williams etal. [31]

\section{Conclusion}

From what we can conclude from the comparison with other populations is that Sudanese renal characters differ from the other populations. A new chart for renal character and measurement were established for Sudanese population aged from 10 years to 70 years

\section{Acknowledgements}

We sincerely thank the participants without whom the study would not have been feasible. The Sudan University of Science and Technology, College of Medical Radiological Science and Radiology Departments are thankfully acknowledged.

\section{References}

[1]. Buchholz NP, Abbas F, Biyabani SR, Afzal M, Javed Q, Rizvi I, et al., 2000. Ultrasonographic renal size in individuals without known renal disease. J. Pak Med Assoc. 50: 12-16.

[2]. $\quad$ E. Supriyanto., Nurul A.T., Syed M.N., Automatic Ultrasound Kidney's Centroid Detection System, 15th International WSEAS GSCC Multiconferences held in Corfu Island, Greece, July 14-16, 2011 ISBN: 978-1-61804-019-0 Page 160-16

[3]. Adeela Arooj, Jostinah Lam, Yeoh J.Wui, Eko Supriyanto, Comparison Of Renal Size Among Different Ethnicities International Journal Of Biology And Biomedical Engineering Issue 4, Volume 5, 2011,221-229

[4]. Kasper DL, Braunwald E, Fauci AS. 16th ed. New York: McGraw Hill; 2005. Harrison's principles of internal medicine; pp. 165363.

[5]. Stone JA, Xu X, Winchell GA, Deutsch PJ, Pearson PG, Migoya EM, et al. Disposition of caspofungin: Role of distribution in determining pharmacokinetics in plasma. Antimicrob Agents Chemother. 2004;48:815-23.

[6]. Sanusi AA, Arogundade FA, Famurewa OC, Akintomide AO, Soyinka FO, Ojo OE, et al. Relationship of ultrasonographically determined kidney volume with measured GFR, calculated creatinine clearance and other parameters in chronic kidney disease (CKD) Nephrol Dial Transplant. 2009;24:1690-4.

[7]. Davidovits M, Eisenstein B, Ziv N, Krause I, Cleper R, Bar-Sever Z. Unilateral duplicated system: Comparative length and function of the kidneys. Clin Nucl Med. 2004; 29:99-102.

[8]. Alexander MP, Patel TV, Farag YM, Florez A, Rennke HG, Singh AK. Kidney pathological changes in metabolic disorder. Am J Kidney Dis. 2009;53:751-9.

[9]. Glodny B, Unterholzner V, Taferner B, Hofmann KJ, Rehder P, Strasak A, et al. Normal kidney size and its influencing factors-a 64 slice MDCT study of 1040 asymptomatic patients. BMC Urol. 2009;9:19.

[10]. Elkin M, 1980. Kidney size, In: Elkin M (ed.), Radiology of the urinary system. Boston, Little, Brown and company: 1014-1032.

[11]. J.J. Chen, Jizu Zhi, Wenyang Mao and G.F. Steinhardt, 2006. MrNomogram: A web-based multivariable pediatric renal nomogram. Journal of Paediatric Radiology.Vol. 2 (5): 436-438.

[12]. J.J. Chen, J. Pugach, M. Patel, A. Luisiri and G.F. Steinhardt, 2002. The renal length nomogram: a multivariate approach. J Urology. Vol. 168: 2149-2152

[13]. Justo Oyuela-Carrasco, Franciso Rodriguez-Castellanos, Eric Kimura, Rosa Elena Delgado, Juan Pablo Herrera-Felix, 2009. Renal length by ultrasound in Mexican adults. Nefrologia. 29(1): 30-34.

[14]. MXrio M. R. Fernandes, Carla C.S.Lemos, Guilherme S. Lopes, Eugenio P.Q. Madeira, Omar R. Santos, David Dorigo, Raquel Bregman, 2002. Normal renal dimensions in a specific population. International Braz J Urol.Vol. 28 (6): 510-515.

[15]. Mazzotta L, Sarteschi LM, Carlini A, Antonelli A., 2002. Comparison of renal ultrasonographic and functional biometry in healthy patients and in patients with chronic renal failure. Arch Ital Urol Androl. 74(4): 206- 209

[16]. Odita JC, 1982. Roentgenologic estimation of kidney in adult Nigerians.Trop Geogr Med. 34: 177-181.

[17]. J.M. Zerin and C.E. Blane, 1994. Sonographic evaluation of renal length in children: a reappraisal. Pediatric Radiology. Vol. 24: $101-106$

[18]. J.M. Zerin and C.E. Blane, 1994. Sonographic evaluation of renal length in children: a reappraisal. Pediatric Radiology. Vol. 24 : $101-106$.

[19]. Justo Oyuela-Carrasco, Franciso Rodriguez-Castellanos, Eric Kimura, IRosa Elena Delgado, Juan Pablo Herrera-Felix, 2009. Renal length by ultrasound in Mexican adults. Nefrologia. 29(1): 30-34.

[20]. MXrio M. R. Fernandes, Carla C.S.Lemos, Guilherme S. Lopes, EugenioP.Q. Madeira, Omar R. Santos, David Dorigo, Raquel Bregman, 2002.Normal renal dimensions in a specific population. International Braz J Urol.Vol. 28 (6): 510-515.

[21]. Mazzotta L, Sarteschi LM, Carlini A, Antonelli A., 2002. Comparison ofrenal ultrasonographic and functional biometry in healthy patients and in patients with chronic renal failure. Arch Ital Urol Androl. 74(4): 206- 209.

[22]. Seyed Alireza Emamian, Michael Bachmann Nielsen, Jan Fog Pedersen,Lars Ytte, 1993. Kidney dimensions at sonography: Correlation with age,sex and habitus in 665 adult volunteers. AJR. 160: 83-86.

[23]. Wing Hang Luk, Adrian Xu Ning Lo, Andrea Wai San Au-Yeung,KarenKa Yee Liu, Yip Hin Woo, Chilene Chi Lin Chiang, Kitty Kit Lin Lo,2010. Renal length nomogram in Hong Kong Asian children:Sonographic measurement and multivariable approach. Journal of Paedaitrics \& Child Health. Vol. 46 (6): 310-315

[24]. Justo Oyuela-Carrasco, Franciso Rodriguez-Castellanos, Eric Kimura, Rosa Elena Delgado, Juan Pablo Herrera-Felix, 2009. Renal length by ultrasound in Mexican adults. Nefrologia. 29(1): 30-34. 
[25]. Wing Hang Luk, Adrian Xu Ning Lo, Andrea Wai San Au-Yeung, Karen Ka Yee Liu, Yip Hin Woo, Chilene Chi Lin Chiang, Kitty Kit Lin Lo, 2010. Renal length nomogram in Hong Kong Asian children: Sonographic measurement and multivariable approach. Journal of Paedaitrics \& Child Health. Vol. 46 (6): 310-315

[26]. Emamian SA, Nielsen MB, Pedersen JF, Ytte L. Kidney dimensions at sonography: Correlation with age, sex and habitus in 665 adult volunteers. AJR Am J Roentgenol 1993;160:83-6.

[27]. J. Oyuela-Carrasco, F. Rodriguez-Castellanos, E. Kimura, R. Delgado- Hernandez, J.P. Herrera-Felix. Renal length by ultrasound in Mexican adults. Nefrologia 20009;29(1):30-34

[28]. Okoye I.J., Aqwu K.K, Indigo FU. Normal sonographic renal length in adult southeast Nigerians. Afr J Med Med Sci.2005 Jun; 34(2):129-31

[29]. D. Shani, I. Jit, L. Sodhi, Weight and Measurements of kidneys in Northwest Indian adults. American Journal of Human Biology 2001,13:726-732.

[30]. Tanaka G, Nakahara Y, Nakazima Y. 1989. Japanese Reference Man 1988 - 1V studies on the weight and size of internal organs of normal Japanese. Nippon Acta Radiol $49: 344-364$.

[31]. Williams PL, Bannister LH, Berry MM, Collins P, Dyson M, Dussek JE, Ferguson MWJ. 1995. Gray's Anatomy, 38th edition.London :Churchill Livingston. 